\title{
ON DEEP HOLES OF STANDARD REED-SOLOMON CODES
}

\author{
RONGJUN WU AND SHAOFANG HONG*
}

\begin{abstract}
Determining deep holes is an important open problem in decoding ReedSolomon codes. It is well known that the received word is trivially a deep hole if the degree of its Lagrange interpolation polynomial equals the dimension of the ReedSolomon code. For the standard Reed-Solomon codes $[p-1, k]_{p}$ with $p$ a prime, Cheng and Murray conjectured in 2007 that there is no other deep holes except the trivial ones. In this paper, we show that this conjecture is not true. In fact, we find a new class of deep holes for standard Reed-Solomon codes $[q-1, k]_{q}$ with $q$ a prime power of $p$. Let $q \geq 4$ and $2 \leq k \leq q-2$. We show that the received word $u$ is a deep hole if its Lagrange interpolation polynomial is the sum of monomial of degree $q-2$ and a polynomial of degree at most $k-1$. So there are at least $2(q-1) q^{k}$ deep holes if $k \leq q-3$.
\end{abstract}

\section{Introduction and the statement of the main result}

Let $\mathbf{F}_{q}$ be the finite field of $q$ elements with characteristic $p$. Let $D=\left\{x_{1}, \ldots, x_{n}\right\}$ be a subset of $\mathbf{F}_{q}$, which is called the evaluation set. The generalized Reed-Solomon code $\mathcal{C}_{q}(D, k)$ of length $n$ and dimension $k$ over $\mathbf{F}_{q}$ is defined as follows:

$$
\mathcal{C}_{q}(D, k)=\left\{\left(f\left(x_{1}\right), \ldots, f\left(x_{n}\right)\right) \in \mathbf{F}_{q}^{n} \mid f(x) \in \mathbf{F}_{q}[x], \operatorname{deg} f(x) \leq k-1\right\} .
$$

If $D=\mathbf{F}_{q}^{*}$, it is called standard Reed-Solomon code. We refer the above definition as the polynomial code version of the standard Reed-Solomon code. If $D=\mathbf{F}_{q}$, it is called extended Reed-Solomon code. For any $[n, k]_{q}$ linear code $\mathcal{C}$, the minimum distance $d(\mathcal{C})$ is defined by

$$
d(\mathcal{C}):=\min \{d(x, y) \mid x \in \mathcal{C}, y \in \mathcal{C}, x \neq y\},
$$

where $d(\cdot, \cdot)$ denotes the Hamming distance of two words which is the number of different entries of them and $w(\cdot)$ denotes the Hamming weight of a word which is the number of its non-zero entries. Thus we have

$$
d(\mathcal{C})=\min \{d(x, 0) \mid 0 \neq x \in \mathcal{C}\}=\min \{w(x) \mid 0 \neq x \in \mathcal{C}\} .
$$

The error distance to code $\mathcal{C}$ of a received word $u \in \mathbf{F}_{q}^{n}$ is defined by $d(u, \mathcal{C}):=$ $\min \{d(u, v) \mid v \in \mathcal{C}\}$. Clearly $d(u, \mathcal{C})=0$ if and only if $u \in \mathcal{C}$. The covering radius $\rho(\mathcal{C})$ of code $\mathcal{C}$ is defined to be $\max \left\{d(u, \mathcal{C}) \mid u \in \mathbf{F}_{p}^{n}\right\}$. For generalized Reed-Solomon code $\mathcal{C}=\mathcal{C}_{q}(D, k)$, we have that the minimum distance $d(\mathcal{C})=n-k+1$ and the covering radius $\rho(\mathcal{C})=n-k$. The most important algorithmic problem in coding theory is the maximum likelihood decoding (MLD): Given a received word, find a word $v \in \mathcal{C}$ such that $d(u, v)=d(u, \mathcal{C})[5]$. Therefore, it is very crucial to decide $d(u, \mathcal{C})$ for the word $u$.

Date: November 5, 2018.

2000 Mathematics Subject Classification. Primary 11Y16, 11T71, 94B35, 94B65.

Key words and phrases. Deep hole, Reed-solomon code, Cyclic code, BCH code, DFT, IDFT.

*Corresponding author and was supported partially by National Science Foundation of China Grant \# 10971145 and by the Ph.D. Programs Foundation of Ministry of Education of China Grant \#20100181110073. 
Sudan 8] and Guruswami-Sudan [2] provided a polynomial time list decoding algorithm for the decoding of $u$ when $d(u, \mathcal{C}) \leq n-\sqrt{n k}$. When the error distance increases, the decoding becomes NP-complete for generalized Reed-Solomon codes $[3$.

When decoding the generalized Reed-Solomon $\operatorname{code} \mathcal{C}$, for a received word $u=\left(u_{1}, \ldots, u_{n}\right)$ $\in \mathbf{F}_{q}^{n}$, we define the Lagrange interpolation polynomial $u(x)$ of $u$ by

$$
u(x):=\sum_{i=1}^{n} u_{i} \prod_{\substack{j=1 \\ j \neq i}}^{n} \frac{x-x_{j}}{x_{i}-x_{j}} \in \mathbf{F}_{q}[x],
$$

i.e., $u(x)$ is the unique polynomial of degree at most $n-1$ such that $u\left(x_{i}\right)=u_{i}$ for $1 \leq i \leq n$. For $u \in \mathbf{F}_{q}^{n}$, we define the degree of $u(x)$ to be the degree of $u$, i.e., $\operatorname{deg}(u)=\operatorname{deg}(u(x))$. It is clear that $d(u, \mathcal{C})=0$ if and only if $\operatorname{deg}(u) \leq k-1$. Evidently, we have the following simple bounds.

Lemma 1.1. 4. For $k \leq \operatorname{deg}(u) \leq n-1$, we have the inequality

$$
n-\operatorname{deg}(u) \leq d(u, \mathcal{C}) \leq n-k=\rho .
$$

Let $u \in \mathbf{F}_{q}^{n}$. If $d(u, \mathcal{C})=n-k$, then the word $u$ is called a deep hole. If $\operatorname{deg}(u)=k$, then the upper bound is equal to the lower bound, and so $d(u, \mathcal{C})=n-k$ which implies that $u$ is a deep hole. This immediately gives $(q-1) q^{k}$ deep holes. We call these deep holes the trivial deep holes. It is an interesting open problem to determine all deep holes. Cheng and Murray 11 showed that for the standard Reed-Solomon code $[p-1, k]_{p}$ with $k<p^{1 / 4-\epsilon}$, the received vector $(f(\alpha))_{\alpha \in \mathbf{F}_{p}^{*}}$ cannot be a deep hole if $f(x)$ is a polynomial of degree $k+d$ for $1 \leq d<p^{3 / 13-\epsilon}$. Based on this result they conjectured that there is no other deep holes except the trivial ones mentioned above. Li and Wan 5 use the method of character sums to obtain a bound on the non-existence of deep holes for extended Reed-Solomon code $\mathcal{C}_{q}\left(\mathbf{F}_{q}, k\right)$.

In this paper, we introduce a new method to investigate the standard Reed-Solomon code $\mathcal{C}_{q}\left(\mathbf{F}_{q}^{*}, k\right)$ and particularly we study its deep holes. In fact, we use the BCH code and the discrete Fourier transform to find some new deep holes. We have the following result.

Theorem 1.2. Let $q \geq 4$ and $2 \leq k \leq q-2$. If the Lagrange interpolation polynomial of a received word $u \in \mathbf{F}_{q}^{q-1}$ is of the form either ax $x^{q-2}+f_{\leq k-1}(x)$ or ax $x^{k}+f_{\leq k-1}(x)$, where $a \in \mathbf{F}_{q}^{*}$ and $f_{\leq k-1}(x) \in \mathbf{F}_{q}[x]$ is of degree at most $k-1$, then $u$ is a deep hole of the standard Reed-Solomon code $\mathcal{C}_{q}\left(\mathbf{F}_{q}^{*}, k\right)$.

If one picks $k=q-2$, then the deep holes given by Theorem 1.2 are the same as the trivial ones. If $k<q-2$, then there exist two types deep holes, namely, we give $2(q-1) q^{k}$ deep holes. Theorem 1.2 also implies that the Cheng-Murray conjecture [1] is not true.

This paper is organized as follows. First we give deep holes for the cyclic code version of the standard Reed-Solomon codes in Section 2. Subsequently, in Section 3, by using the results presented in Section 2 and the discrete Fourier transformation of vectors, we show Theorem 1.2. Finally, we give in Section 4 some examples of the received word $u$ which is not a deep hole and whose Lagrange interpolation polynomial is of the form neither $a x^{q-2}+f_{\leq k-1}(x)$ nor $a x^{k}+f_{\leq k-1}(x)$. We suggest a conjecture on the nonexistence of deep holes for the standard Reed-Solomon code $\mathcal{C}_{q}\left(\mathbf{F}_{q}^{*}, k\right)$ at the end of the paper. 


\section{Deep hole of the standard Reed-Solomon codes as cyclic codes}

In the present section, we consider the deep hole for the cyclic code version of the standard Reed-Solomon codes. Throughout this paper, we let $\alpha$ be a fixed primitive element of $\mathbf{F}_{q}$ and

$$
g(x)=(x-\alpha)\left(x-\alpha^{2}\right) \ldots\left(x-\alpha^{d-1}\right) .
$$

We define the standard Reed-Solomon code with length $n=q-1$ as follows:

$$
\mathcal{C}:=\left\{g(x) m(x) \in \mathbf{F}_{q}[x] \mid m(x) \in \mathbf{F}_{q}[x] \text { and } \operatorname{deg}(m(x)) \leq k-1\right\} .
$$

and refer it as the cyclic code version of the standard Reed-Solomon code. It is a maximal distance separable code, i.e., we have $d=n-k+1=q-k$.

Now let $D=\mathbf{F}_{q}^{*}=\left\{1, \alpha, \ldots, \alpha^{q-2}\right\}$. Then the the polynomial code version of the standard Reed-Solomon code can be stated as follows

$$
\mathcal{C}_{q}\left(\mathbf{F}_{q}^{*}, k\right):=\left\{\left(f(1), f(\alpha), \ldots, f\left(\alpha^{q-2}\right)\right) \in \mathbf{F}_{q}^{n} \mid f(x) \in \mathbf{F}_{q}[x], \operatorname{deg} f(x) \leq k-1\right\} .
$$

Let's recall the definition of $\mathrm{BCH}$ code and the $\mathrm{BCH}$ bound.

Definition 2.1. 6] Let $\delta \geq 2$ be an integer. A cyclic code of length $n$ over $\mathbf{F}_{q}$ is called a $B C H$ code of designed distance $\delta$ if its generator $g(x)$ is the least common multiple of the minimal polynomials of $\beta^{l}, \beta^{l+1}, \ldots, \beta^{l+\delta-2}$ for some positive integer $l$, where $\beta$ is a primitive $n$th root of unity. If $n=q^{m}-1$ for some integer $m \geq 1$, then the $\mathrm{BCH}$ code is called primitive.

The designed distance is explained by the following result which is usually called $B C H$ bound.

Lemma 2.2. 6] The minimum distance of a $B C H$ code with designed distance $\delta$ is at least $\delta$.

We are now in a position to give the main result of this section.

Theorem 2.3. For the cyclic code version of the standard Reed-Solomon code $\mathcal{C}$ defined as in (2.1), let $g_{1}(x):=g(x) /(x-\alpha)$. Then $a g_{1}(x)+l(x) g(x)$ is a deep hole of $\mathcal{C}$ for any $a \in \mathbf{F}_{q}^{*}$ and $l(x) \in \mathbf{F}_{q}[x]$ with $\operatorname{deg}(l(x)) \leq k-1$.

Proof. First we show that $g_{1}(x)$ is a deep hole. For any codeword $m(x) g(x) \in \mathcal{C}$ with $\operatorname{deg}(m(x)) \leq k-1$, we have

$$
d\left(g_{1}(x), m(x) g(x)\right)=w\left(g_{1}(x)(1-m(x)(x-\alpha))\right)
$$

where $w(\cdot)$ means the number of the nonzero coefficients of the polynomial. Let

$$
\Delta(x):=1-m(x)(x-\alpha) .
$$

We define the primitive $\mathrm{BCH}$ code $C^{\prime}$ as follows:

$$
\mathcal{C}^{\prime}=\left\{c(x)=\sum_{i=0}^{q-2} c_{i} x^{i} \in \mathbf{F}_{q}[x] /\left(x^{q-1}-1\right) \mid c\left(\alpha^{2}\right)=\ldots=c\left(\alpha^{d-1}\right)=0\right\} .
$$

Then the designed distance $\delta$ of $\mathcal{C}^{\prime}$ is $d-1$ since its generator, the least common multiple of the minimal polynomials of $\alpha^{2}, \ldots, \alpha^{d-1}$, is $g_{1}(x)$. For the BCH code, by Lemma 2.2 we know that the minimum distance $d^{\prime}$ is at least $\delta$. Since $\operatorname{deg}(\Delta(x)) \leq k$ and $d=q-k$, we have $\operatorname{deg}\left(g_{1}(x) \Delta(x)\right) \leq q-2$. But $g_{1}\left(\alpha^{i}\right)=0$ for $2 \leq i \leq d-1$. So $g_{1}(x)(\Delta(x)) \in \mathcal{C}^{\prime}$. Note 
that $\mathcal{C}^{\prime}$ holds minimum distance $d^{\prime} \geq d-1$. Therefore $w\left(g_{1}(x) \Delta(x)\right) \geq d-1=q-1-k$. It then follows that $g_{1}(x)$ is a deep hole.

Subsequently, we compute the distance between $a g_{1}(x)+l(x) g(x)$ and the standard Reed-Solomon codes $\mathcal{C}$. Since $a \in \mathbf{F}_{q}^{*}$ and $l(x) \in \mathbf{F}_{q}[x]$, we infer that

$$
\begin{aligned}
& d\left(a g_{1}(x)+l(x) g(x), \mathcal{C}\right) \\
& =\min \left\{d\left(a g_{1}(x)+l(x) g(x), m(x) g(x)\right) \mid m(x) \in \mathbf{F}_{q}[x] \text { and } \operatorname{deg}(m(x)) \leq k-1\right\} \\
& =\min \left\{d\left(a g_{1}(x)+l(x) g(x),(m(x)+l(x)) g(x)\right) \mid m(x) \in \mathbf{F}_{q}[x] \text { and } \operatorname{deg}(m(x)) \leq k-1\right\} \\
& =\min \left\{d\left(a g_{1}(x), m(x) g(x)\right) \mid m(x) \in \mathbf{F}_{q}[x] \text { and } \operatorname{deg}(m(x)) \leq k-1\right\} \\
& =\min \left\{d\left(a g_{1}(x), a m(x) g(x)\right) \mid m(x) \in \mathbf{F}_{q}[x] \text { and } \operatorname{deg}(m(x)) \leq k-1\right\} \\
& =\min \left\{d\left(g_{1}(x), m(x) g(x)\right) \mid m(x) \in \mathbf{F}_{q}[x] \text { and } \operatorname{deg}(m(x)) \leq k-1\right\} \\
& =d\left(g_{1}(x), \mathcal{C}\right)=q-1-k=n-k .
\end{aligned}
$$

Therefore $a g_{1}(x)+l(x) g(x)$ is a deep hole. This completes the proof of Theorem 2.3.

By the similar argument as in the proof of Theorem 2.3, one can deduce the following result.

Theorem 2.4. Let $g_{2}(x)=g(x) /\left(x-\alpha^{d-1}\right)$. Then $a g_{2}(x)+l(x) g(x)$ is a deep hole of $\mathcal{C}$ for any $a \in \mathbf{F}_{q}^{*}$ and $l(x) \in \mathbf{F}_{q}[x]$ with $\operatorname{deg}(l(x)) \leq k-1$.

It is natural to ask: Is there any other deep holes except those in Theorems 2.3 and 2.4? We believe the answer to this question should be negative if the characteristic of $\mathbf{F}_{q}$ is an odd prime, but we have not yet found a proof. In the next section, by discrete Fourier transformation, and Theorem 2.3 we will arrive at the deep holes of the standard Reed-Solomon code for the polynomial code version.

\section{Proof of Theorem 1.2}

In this section, we first recall the definitions of the discrete Fourier transform (DFT) and inverse discrete Fourier transform (IDFT) of vectors over $\mathbf{F}_{q}$ (see, for example, [7]). Then we use them to give the relationship between the polynomial code version and cyclic code version of the standard Reed-Solomon codes. Let $\alpha$ be the fixed primitive element of $\mathbf{F}_{q}$. Let $V=\left(V_{0}, V_{1}, \ldots, V_{q-2}\right) \in \mathbf{F}_{q}^{q-1}$. The discrete Fourier transform (DFT) of $V$, denoted by $\hat{V}=\left(\hat{V}_{0}, \hat{V}_{1}, \ldots, \hat{V}_{q-2}\right)$, is defined as follows:

$$
\hat{V}_{j}:=\sum_{i=0}^{q-2} V_{i} \alpha^{i j}
$$

for $j=0,1, \ldots, q-2$. We also call $V$ the inverse discrete Fourier transform (IDFT) of $\hat{V}$. Sometimes we call the $V_{i}$ 's the "time-domain" coordinates of the vector $V$, and the $\hat{V}_{j}$ 's the "frequency-domain" coordinates, of the vector $V$. The time-domain components can be recovered from the frequency-domain components via the IDFT:

$$
V_{i}=\frac{1}{q-1} \sum_{j=0}^{q-2} \hat{V}_{j} \alpha^{-i j}
$$


for $i=0,1, \ldots, q-2$. If we interpret the components of the $V$ and $\hat{V}$ as the coefficients of polynomials, i.e., if we define generating functions $V(x)$ and $\hat{V}(x)$ by

$$
V(x)=V_{0}+V_{1} x+\cdots+V_{q-2} x^{q-2}
$$

and

$$
\hat{V}(x)=\hat{V}_{0}+\hat{V}_{1} x+\cdots+\hat{V}_{q-2} x^{q-2},
$$

then the DFT and IDFT relationships (3.1) and (3.2) can be rewritten in the following:

$$
\hat{V}_{j}=V\left(\alpha^{j}\right)
$$

and

$$
V_{i}=\frac{1}{q-1} \hat{V}\left(\alpha^{-i}\right)
$$

We call $\hat{V}(x)$ the DFT of $V(x)$ and $V(x)$ the IDFT of $\hat{V}(x)$. Evidently, the fact $\hat{V}(x)$ is the DFT of $V(x)$ implies that $V(x)$ is the IDFT of $\hat{V}(x)$, and vise versa. Note that if $\widehat{V^{(1)}}$ and $\widehat{V^{(2)}}$ are the DFT of $(q-1)$-dimensional vectors $V^{(1)}$ and $V^{(2)}$ over $\mathbf{F}_{q}$ respectively, then $\lambda \widehat{V^{(1)}}+\mu \widehat{V^{(2)}}$ is the DFT of $\lambda V^{(1)}+\mu V^{(2)}$ for any $\lambda \in \mathbf{F}_{q}$ and $\mu \in \mathbf{F}_{q}$. Note also that if $V^{(1)}$ and $V^{(2)}$ are the IDFT of $(q-1)$-dimensional vectors $\widehat{V^{(1)}}$ and $\widehat{V^{(2)}}$ over $\mathbf{F}_{q}$ respectively, then $\lambda V^{(1)}+\mu V^{(2)}$ is the IDFT of $\lambda \widehat{V^{(1)}}+\mu \widehat{V^{(2)}}$ for any $\lambda \in \mathbf{F}_{q}$ and $\mu \in \mathbf{F}_{q}$. In other words, the DFT and the IDFT are linear operations in $\mathbf{F}_{q}^{q-1}$.

There is a one-to-one correspondence of codewords between the cyclic code version and polynomial code version of the standard Reed-Solomon code as the following lemma shows.

Lemma 3.1. (1). For any $s(x) \in \mathbf{F}_{q}[x]$ with $\operatorname{deg}(s(x)) \leq k-1$, there exists an $l(x) \in$ $\mathbf{F}_{q}[x]$ with $\operatorname{deg}(l(x)) \leq k-1$ such that $l(x) g(x)$ is the DFT of $s(x)$.

(2). For any $l(x) \in \mathbf{F}_{q}[x]$ with $\operatorname{deg}(l(x)) \leq k-1$, there exists an $s(x) \in \mathbf{F}_{q}[x]$ with $\operatorname{deg}(s(x)) \leq k-1$ such that $s(x)$ is the IDFT of $l(x) g(x)$.

Proof. (1). Write $s(x)=\sum_{i=0}^{k-1} s_{i} x^{i}$ and let $\hat{s}(x)=\sum_{j=0}^{q-2} \hat{s_{j}} x^{j}$ be the DFT of $s(x)$. By (3.3) we have

for $0 \leq j \leq q-2$. Thus

$$
\hat{s_{j}}=s\left(\alpha^{j}\right)
$$

$$
\hat{s}(x)=\sum_{j=0}^{q-2} s\left(\alpha^{j}\right) x^{j} .
$$

Since $g(x)=(x-\alpha)\left(x-\alpha^{2}\right) \cdots\left(x-\alpha^{d-1}\right), \operatorname{deg} \hat{s}(x) \leq q-2$ and $k-1=q-2-(d-1)$, in order to prove that there is an $l(x) \in \mathbf{F}_{q}[x]$ with $\operatorname{deg}(l(x)) \leq k-1$ such that $\hat{s}(x)=$ $l(x) g(x)$, it is sufficient to show that if $1 \leq m \leq d-1$, then $\hat{s}\left(\alpha^{m}\right)=0$. By (3.5) we have

$$
\begin{aligned}
\hat{s}\left(\alpha^{m}\right) & =\sum_{j=0}^{q-2} s\left(\alpha^{j}\right) \alpha^{m j} \\
& =\sum_{j=0}^{q-2} \sum_{i=0}^{k-1} s_{i} \alpha^{j i} \alpha^{m j} \\
& =\sum_{i=0}^{k-1} s_{i} \sum_{j=0}^{q-2} \alpha^{(m+i) j} .
\end{aligned}
$$


Since $d=q-k$, one infers that $1 \leq m+i \leq d+k-2=q-2$ for $0 \leq i \leq k-1$ and $1 \leq m \leq d-1$. This implies that $\alpha^{m+i} \neq 1$. Since $\alpha$ is the primitive root of $\mathbf{F}_{q}$, it follows immediately that $\sum_{j=0}^{q-2} \alpha^{(m+i) j}=0$. Hence by (3.6), we get that $\hat{s}\left(\alpha^{m}\right)=0$ for $1 \leq m \leq d-1$ as required. Part (1) is proved.

(2). Let $s(x)=\sum_{i=0}^{q-2} s_{i} x^{i}$ be the IDFT of $l(x) g(x)$. Then by (3.4) we have

$$
s_{i}=\frac{1}{q-1} l\left(\alpha^{-i}\right) g\left(\alpha^{-i}\right)=\frac{1}{q-1} l\left(\alpha^{-i}\right) g\left(\alpha^{q-1-i}\right)
$$

for $0 \leq i \leq q-2$. But $g(x)=\prod_{i=k}^{q-2}\left(x-\alpha^{q-1-i}\right)$. So we have $g\left(\alpha^{q-1-i}\right)=0$ for $k \leq i \leq q-2$. One then derives that $s_{i}=0$ for $k \leq i \leq q-2$. That is, $\operatorname{deg}(s(x)) \leq k-1$ as desired. Part (2) is proved.

The proof of Lemma 3.1 is complete.

Consequently we describe the DFT of $u(x)=\sum_{i=0}^{q-2} u_{i} x^{i} \in \mathbf{F}_{q}[x]$ with $u_{q-2} \neq 0$ and $u_{i}=0$ for all $k \leq i \leq q-3$ in the following lemma.

Lemma 3.2. Let $u(x)=\sum_{i=0}^{q-2} u_{i} x^{i} \in \mathbf{F}_{q}[x]$ satisfy $u_{q-2} \neq 0$ and $u_{i}=0$ for all $k \leq i \leq q-3$. Then there exist $a \in \mathbf{F}_{q}^{*}$ and $l(x) \in \mathbf{F}_{q}[x]$ with degree $\leq k-1$ such that $a g_{1}(x)+l(x) g(x)$ is the DFT of $u(x)$.

Proof. Let

$$
\hat{V}(x)=g_{1}(x)=\left(x-\alpha^{q-1-(q-3)}\right)\left(x-\alpha^{q-1-(q-4)}\right) \cdots\left(x-\alpha^{q-1-k}\right)
$$

and write its IDFT as

$$
V(x)=V_{0}+V_{1} x+\cdots+V_{q-2} x^{q-2} .
$$

Then

$$
V_{i}=\frac{1}{q-1} \hat{V}\left(\alpha^{q-1-i}\right)=\frac{1}{q-1} g_{1}\left(\alpha^{q-1-i}\right)
$$

for $i=0,1, \ldots, q-2$. For $i=k, \ldots, q-3$, since $\alpha^{q-1-i}$ is a zero of $g_{1}(x)$, we have $V_{i}=0$. But

$$
V_{q-2}=\frac{1}{q-1} g_{1}(\alpha)=\frac{1}{q-1} \prod_{j=k}^{q-3}\left(\alpha-\alpha^{q-1-j}\right) \neq 0 .
$$

Thus the IDFT of $g_{1}(x)$ is

$$
V(x)=V_{q-2} x^{q-2}+V_{k-1} x^{k-1}+V_{k-2} x^{k-2}+\cdots+V_{1} x+V_{0},
$$

where $V_{q-2} \neq 0$.

On the other hand, by Lemma 3.1 (1) there exist $l_{1}(x) \in \mathbf{F}_{q}[x]$ and $l_{2}(x) \in \mathbf{F}_{q}[x]$ with $\operatorname{deg}\left(l_{1}(x)\right) \leq k-1$ and $\operatorname{deg}\left(l_{2}(x)\right) \leq k-1$ such that $l_{1}(x) g(x)$ equals the DFT of $\sum_{i=0}^{k-1} V_{i} x^{i}$ and $l_{2}(x) g(x)$ is equal to the DFT of $\sum_{i=0}^{k-1} u_{i} x^{i}$. Since the DFT and IDFT are linear operations in $\mathbf{F}_{q}^{q-1}$, it follows that the IDFT of $g_{1}(x)-l_{1}(x) g(x)$ is $V_{q-2} x^{q-2}$.

Now let $a=\frac{u_{q-2}}{V_{q-2}} \in \mathbf{F}_{q}^{*}$ and $l(x)=-a l_{1}(x)+l_{2}(x)$. Then $\operatorname{deg} l(x) \leq k-1$. One can deduce that

$$
\text { the IDFT of } a g_{1}(x)-a l_{1}(x) g(x)=u_{q-2} x^{q-2} .
$$

Hence

$$
a g_{1}(x)+l(x) g(x)=\text { the DFT of } u_{q-2} x^{q-2}+\sum_{i=0}^{k-1} u_{i} x^{i}
$$

as required. Lemma 3.2 is proved. 
Remark. Similarly, one can prove that there exist an $a \in \mathbf{F}_{q}^{*}$ and an $l(x) \in \mathbf{F}_{q}[x]$ with degree $\leq k-1$ such that $a g_{2}(x)+l(x) g(x)$ is the DFT of $x^{k}$.

Next, we show that the DFT is distance preserved.

Lemma 3.3. Let $s(x), t(x) \in \mathbf{F}_{q}[x]$ be of degree at most $q-2, s=\left(s(1), s(\alpha), \ldots, s\left(\alpha^{q-2}\right)\right)$ and $t=\left(t(1), t(\alpha), \ldots, t\left(\alpha^{q-2}\right)\right)$. Let $\hat{s}(x)$ and $\hat{t}(x)$ be the DFT of $s(x)$ and $t(x)$ respectively. Then we have

$$
d(s, t)=d(\hat{s}(x), \hat{t}(x)) .
$$

Proof. Since $d(s, t)=w(s-t)$ and $d(\hat{s}(x), \hat{t}(x))=w(\hat{s}(x)-\hat{t}(x))$, in order to prove that $d(s, t)=d(\hat{s}(x), \hat{t}(x))$, it suffices to show that

$$
w(s-t)=w(\hat{s}(x)-\hat{t}(x)) .
$$

Note that $s-t=\left(s(1)-t(1), s(\alpha)-t(\alpha), \ldots, s\left(\alpha^{q-2}\right)-t\left(\alpha^{q-2}\right)\right)$. So one can easily check that

$$
w(s-t)=q-1-\#\left\{\text { ditinct roots in } \mathbf{F}_{q}^{*} \text { of } s(x)-t(x)=0\right\} .
$$

Thus one needs only to show that

$$
w(\hat{s}(x)-\hat{t}(x))=q-1-\#\left\{\text { ditinct roots in } \mathbf{F}_{q}^{*} \text { of } s(x)-t(x)=0\right\} .
$$

Let $\hat{v}(x)=\hat{v}_{0}+\hat{v}_{1} x+\ldots+\hat{v}_{q-2} x^{q-2}=\hat{s}(x)-\hat{t}(x) \in \mathbf{F}_{q}[x]$. Since $\hat{s}(x)-\hat{t}(x)=\widehat{s-t}(x)$, it follows that $v(x)=s(x)-t(x)$ is the IDFT of $\hat{v}(x)$. Therefore $\hat{v}_{j}=v\left(\alpha^{j}\right)$. Thus to prove (3.7) is equivalent to show the following identity:

$$
w(\hat{v}(x))=q-1-\#\left\{\text { distinct roots in } \mathbf{F}_{q}^{*} \text { of } v(x)=0\right\} .
$$

But we have

$$
\begin{aligned}
w(\hat{v}(x)) & =\#\left\{0 \leq j \leq q-2 \mid \hat{v}_{j} \neq 0\right\} \\
& =\#\left\{0 \leq j \leq q-2 \mid v\left(\alpha_{j}\right) \neq 0\right\} \\
& =q-1-\#\left\{0 \leq j \leq q-2 \mid v\left(\alpha_{j}\right)=0\right\} \\
& =q-1-\#\left\{\text { distinct roots in } \mathbf{F}_{q}^{*} \text { of } v(x)=0\right\} .
\end{aligned}
$$

So (3.8) is proved. This completes the proof of Lemma 3.3.

Finally, we show Theorem 1.2 as follows.

Proof of Theorem 1.2: Write $u(x)=\sum_{i=0}^{q-2} u_{i} x^{i} \in \mathbf{F}_{q}[x]$ as the Lagrange interpolation polynomial of the received word $u$, where either $u_{q-2} \neq 0$ and $u_{i}=0$ for $k \leq i \leq q-3$ or $u_{k} \neq 0$ and $u_{i}=0$ for $k+1 \leq i \leq q-2$. Further, we can write $u=\left(u(1), u(\alpha), \ldots, u\left(\alpha^{q-2}\right)\right) \in \mathbf{F}_{q}^{q-1}$, where $\alpha$ is the fixed primitive element of $\mathbf{F}_{q}$. By Lemma 3.3, we have

$$
\begin{aligned}
d\left(u, \mathcal{C}_{q}\left(\mathbf{F}_{q}^{*}, k\right)\right) & =\min \left\{d(u, v) \mid v \in \mathcal{C}_{q}\left(\mathbf{F}_{q}^{*}, k\right)\right\} \\
& =\min \left\{d(\hat{u}(x), \hat{v}(x)) \mid v \in \mathcal{C}_{q}\left(\mathbf{F}_{q}^{*}, k\right)\right\} .
\end{aligned}
$$

From $v=\left(v(1), v(\alpha), \ldots, v\left(\alpha^{q-2}\right)\right) \in \mathcal{C}_{q}\left(\mathbf{F}_{q}^{*}, k\right)$, we get that $\operatorname{deg}(v(x)) \leq k-1$. Let $\mathcal{C}$ be defined as in (2.1). Then by Lemma 3.1, we have $\hat{v}(x)=l(x) g(x) \in \mathcal{C}$. So

$$
d\left(u, \mathcal{C}_{q}\left(\mathbf{F}_{q}^{*}, k\right)\right)=\min \{d(\hat{u}(x), \hat{v}(x)) \mid \hat{v}(x) \in \mathcal{C}\} .
$$


TABLE 1. The received words for Example 4.1

\begin{tabular}{|c|c|c|c|}
\hline Received word $u$ & Lagrange interpolation polynomial of $u$ & Codeword $v$ & $d(u, v)$ \\
\hline \hline$(8,8,7,8,1,0,0,0,0,0)$ & $1+x+4 x^{2}+3 x^{3}+6 x^{4}+4 x^{8}$ & $(0,8,7,8,8,3,0,0,0,7)$ & 4 \\
\hline$(4,9,5,1,1,0,0,0,0,0)$ & $2+10 x+3 x^{2}+2 x^{3}+8 x^{4}+x^{7}$ & $(0,3,9,9,10,0,8,4,0,0)$ & 4 \\
\hline$(2,3,9,9,1,0,0,0,0,0)$ & $9+2 x+2 x^{2}+10 x^{3}+2 x^{4}+10 x^{6}$ & $(0,3,9,9,10,0,8,4,0,0)$ & 4 \\
\hline
\end{tabular}

On the other hand, by Lemma 3.2 and the remark after Lemma 3.2, we obtain that either $\hat{u}(x)=a g_{1}(x)+l(x) g(x)$ or $\hat{u}(x)=a g_{2}(x)+l(x) g(x)$ for some $a \in \mathbf{F}_{q}^{*}$ and $l(x) \in \mathbf{F}_{q}[x]$ with degree $\leq k-1$. Then we have either

$$
\min \{d(\hat{u}(x), \hat{v}(x)) \mid \hat{v}(x) \in \mathcal{C}\}=\min \left\{d\left(a g_{1}(x)+l(x) g(x), \hat{v}(x)\right) \mid \hat{v}(x) \in \mathcal{C}\right\}
$$

or

$$
\min \{d(\hat{u}(x), \hat{v}(x)) \mid \hat{v}(x) \in \mathcal{C}\}=\min \left\{d\left(a g_{2}(x)+l(x) g(x), \hat{v}(x)\right) \mid \hat{v}(x) \in \mathcal{C}\right\} .
$$

By Theorems 2.3 and 2.4, we know that $a g_{1}(x)+l(x) g(x)$ and $a g_{2}(x)+l(x) g(x)$ are both deep holes of $\mathcal{C}$. Then one can deduce that

$$
\min \{d(\hat{u}(x), \hat{v}(x)) \mid \hat{v}(x) \in \mathcal{C}\}=q-1-k .
$$

Therefore we arrive at $d\left(u, \mathcal{C}_{q}\left(\mathbf{F}_{q}^{*}, k\right)\right)=q-1-k$. Thus $u$ is a deep hole. This complete the proof of Theorem 1.2.

\section{Examples and conjecture}

Let $q \geq 4$ and $2 \leq k \leq q-2$. For any received word $u \in \mathbf{F}_{q}^{q-1} \backslash \mathcal{C}_{q}\left(\mathbf{F}_{q}^{*}, k\right)$, it is clear that the Lagrange interpolation polynomial of $u$ is of degree no less than $k$ and no more than $q-2$. By Theorem 1.2 we know that if the Lagrange interpolation polynomial of the received word $u$ is of the form either $a x^{q-2}+f_{\leq k-1}(x)$ or $a x^{k}+f_{\leq k-1}(x)$, where $a \in \mathbf{F}_{q}^{*}$ and $\operatorname{deg} f_{\leq k-1}(x) \leq k-1$, then $u$ is a deep hole of the standard ReedSolomon code $\mathcal{C}_{q}\left(\mathbf{F}_{q}^{*}, k\right)$. The following two examples tell us that there exist some received words $u$ which are not deep holes and whose Lagrange interpolation polynomials are of the form neither $a x^{q-2}+f_{\leq k-1}(x)$ nor $a x^{k}+f_{\leq k-1}(x)$. In what follows, we let $q=11, n=q-1=10, \alpha=2, d=6, k=5$ and $x_{i}=2^{i-1}$ for $1 \leq i \leq 10$. So we have

$$
\mathcal{C}_{11}\left(\mathbf{F}_{11}^{*}, 5\right)=\left\{\left(f(1), f(2), \ldots, f\left(2^{9}\right)\right) \in \mathbf{F}_{11}^{10} \mid f(x) \in \mathbf{F}_{11}[x], \operatorname{deg} f(x) \leq 4\right\} .
$$

Example 4.1. With received words $u$ as in Table 1 whose Lagrange interpolation polynomial $u(x)=\sum_{i=0}^{q-2} u_{i} x^{i}$ satisfies that $u_{i_{0}} \neq 0$ for exactly one $k+1 \leq i_{0} \leq q-3$ and $u_{i}=0$ for all $k \leq i \leq q-2, i \neq i_{0}$. Using Matlab R2009b, we search and find a codeword $v$ as in Table 1 such that $d(u, v)<n-k=5$. But $d\left(u, \mathcal{C}_{11}\left(\mathbf{F}_{11}^{*}, 5\right)\right) \leq d(u, v)$. Therefore $d\left(u, \mathcal{C}_{11}\left(\mathbf{F}_{11}^{*}, 5\right)\right)<n-k=5$. Namely, the three received words $u$ in Table 1 are not deep holes.

Example 4.2. (1). With received words $u$ as in Table 2 whose Lagrange interpolation polynomial $u(x)=\sum_{i=0}^{q-2} u_{i} x^{i}$ satisfies that $u_{q-2} \neq 0$ and $u_{i_{0}} \neq 0$ for $k \leq i_{0} \leq q-3$. From Table 2, one can read that $d\left(u, \mathcal{C}_{11}\left(\mathbf{F}_{11}^{*}, 5\right)\right) \leq d(u, 0)=w(u) \leq 4<n-k=5$. In other words, the four received words $u$ in Table 2 are not deep holes.

(2). With received words $u$ as in Table 3 whose Lagrange interpolation polynomial $u(x)=\sum_{i=0}^{q-2} u_{i} x^{i}$ satisfies that $u_{q-2} \neq 0, u_{i_{1}} \neq 0$ and $u_{i_{2}} \neq 0$ for $k \leq i_{1} \neq i_{2} \leq q-3$. Using Matlab R2009b, we search and find a codeword $v$ as in Table 3 such that $d(u, v)<$ 
TABLE 2. The received words for Example 4.2 (1)

\begin{tabular}{|c|c|c|}
\hline Received word $u$ & Lagrange interpolation polynomial of $u$ & Weight $w(u)$ \\
\hline \hline$(8,1,2,9,0,0,0,0,0,0)$ & $2+5 x+3 x^{2}+x^{3}+x^{4}+7 x^{8}+9 x^{9}$ & 4 \\
\hline$(1,0,4,5,0,0,0,0,0,0)$ & $1+7 x+4 x^{2}+2 x^{3}+1 x^{4}-x^{7}+9 x^{9}$ & 3 \\
\hline$(3,0,6,0,8,0,0,0,0,0)$ & $5+4 x+5 x^{2}+5 x^{3}+7 x^{4}+x^{6}+9 x^{9}$ & 3 \\
\hline$(4,1,4,3,0,0,0,0,0,0)$ & $-1+x+8 x^{2}+7 x^{3}+6 x^{4}+7 x^{5}+9 x^{9}$ & 4 \\
\hline
\end{tabular}

TABLE 3. The received words for Example 4.2 (2)

\begin{tabular}{|c|c|c|c|}
\hline Received word $u$ & Lagrange interpolation polynomial of $u$ & Codeword $v$ & $d(u, v)$ \\
\hline \hline$(6,4,10,4,3,0,0,0,0,0)$ & $6+6 x+3 x^{2}+9 x^{3}+x^{4}+x^{7}+4 x^{8}+9 x^{9}$ & $(0,4,10,7,3,0,3,0,0,5)$ & 4 \\
\hline$(4,9,3,1,3,0,0,0,0,0)$ & $2+9 x+2 x^{2}+6 x^{3}+6 x^{4}+10 x^{6}+4 x^{8}+9 x^{9}$ & $(2,9,3,7,10,0,0,0,0,10)$ & 4 \\
\hline$(3,3,10,6,3,0,0,0,0,0)$ & $8+1 x+10 x^{2}+4 x^{3}+7 x^{4}+4 x^{5}+4 x^{8}+9 x^{9}$ & $(1,3,10,4,3,0,0,7,0,0)$ & 4 \\
\hline$(0,10,1,5,3,0,0,0,0,0)$ & $3+7 x+x^{2}+5 x^{3}+8 x^{4}+10 x^{6}+x^{7}+9 x^{9}$ & $(0,0,0,0,0,0,0,0,0,0)$ & 4 \\
\hline$(10,4,8,10,3,0,0,0,0,0)$ & $9+10 x+9 x^{2}+3 x^{3}+9 x^{4}+4 x^{5}+x^{7}+9 x^{9}$ & $(1,4,8,10,3,1,0,1,4,0)$ & 4 \\
\hline$(8,9,1,7,3,0,0,0,0,0)$ & $5+2 x+8 x^{2}+3 x^{4}+4 x^{5}+10 x^{6}+9 x^{9}$ & $(2,9,3,7,10,0,0,0,0,10)$ & 4 \\
\hline
\end{tabular}

TABLE 4. The received words for Example 4.2 (3)

\begin{tabular}{|c|c|c|c|}
\hline Received word $u$ & Lagrange interpolation polynomial of $u$ & Codeword $v$ & $d(u, v)$ \\
\hline \hline$(8,7,8,2,4,0,0,0,0,0)$ & $4+8 x+5 x^{2}+8 x^{3}+3 x^{4}+10 x^{6}+x^{7}+4 x^{8}+9 x^{9}$ & $(1,6,8,2,2,0,8,0,0,0)$ & 4 \\
\hline$(7,1,4,7,4,0,0,0,0,0)$ & $10+2 x^{2}+6 x^{3}+4 x^{4}+4 x^{5}+x^{7}+4 x^{8}+9 x^{9}$ & $(1,1,4,7,4,7,8,0,9,0)$ & 4 \\
\hline$(5,6,8,4,4,0,0,0,0,0)$ & $6+3 x+x^{2}+3 x^{3}+9 x^{4}+4 x^{5}+10 x^{6}+4 x^{8}+9 x^{9}$ & $(1,6,8,2,2,0,8,0,0,0)$ & 4 \\
\hline$(1,7,6,8,4,0,0,0,0,0)$ & $7+x+2 x^{3}+4 x^{5}+10 x^{6}+x^{7}+9 x^{9}$ & $(1,9,2,8,4,1,0,0,0,0)$ & 3 \\
\hline
\end{tabular}

$n-k=5$. Therefore $d\left(u, \mathcal{C}_{11}\left(\mathbf{F}_{11}^{*}, 5\right)\right) \leq d(u, v)<n-k=5$. That is, the six received words $u$ in Table 3 are not deep holes.

(3). With received words $u$ as in Table 4 whose Lagrange interpolation polynomial $u(x)=\sum_{i=0}^{q-2} u_{i} x^{i}$ satisfies that $u_{q-2} \neq 0, u_{i_{1}} \neq 0, u_{i_{2}} \neq 0$ and $u_{i_{3}} \neq 0$ for $k \leq i_{1}<i_{2}<$ $i_{3} \leq q-3$. Using Matlab R2009b, we search and find a codeword $v$ as in Table 4 such that $d(u, v)<n-k=5$. It follows that $d\left(u, \mathcal{C}_{11}\left(\mathbf{F}_{11}^{*}, 5\right)\right) \leq d(u, v)<n-k=5$, i.e., the four received words $u$ in Table 4 are not deep holes.

(4). There is received word $u$ whose Lagrange interpolation polynomial $u(x)=\sum_{i=0}^{q-2} u_{i} x^{i}$ satisfies that $u_{q-2} \neq 0$ and $u_{i} \neq 0$ for all $k \leq i \leq q-3$. In fact, let $u=(9,10,4,9,10,0,0,0$, $0,0)$. Then the Lagrange interpolation polynomial of $u$ is $u(x)=2+8 x+5 x^{2}+$ $7 x^{3}+6 x^{4}+7 x^{5}+x^{6}+x^{7}+7 x^{8}+9 x^{9}$. By computervsearching, we find a codeword $v=(1,10,7,9,5,0,0,0,0,5)$ satisfying $d(u, v)=4<n-k$. It follows that $u$ is not a deep hole.

According to the above examples and exhausting computer search, we propose the following conjecture as the conclusion of this paper.

Conjecture 4.3. Assume that the characteristic $p$ of $\mathbf{F}_{q}$ is an odd prime. If the Lagrange interpolation polynomial of the received word $u$ be of the form neither ax $x^{q-2}+f_{\leq k-1}(x)$ nor $a x^{k}+f_{\leq k-1}(x)$, then $u$ is not a deep hole of the standard Reed-Solomon code $\mathcal{C}_{q}\left(\mathbf{F}_{q}^{*}, k\right)$. 
Acknowledgement. The authors would like to thank the anonymous referee for careful reading of the manuscript and for helpful comments and suggestions.

\section{REFERENCES}

[1] Q. Cheng and E. Murray,On deciding deep holes of Reed-Solomon codes, Proceedings of TAMC 2007, LNCS 4484, Berlin: Springer, 296-305.

[2] V. Guruswami and M. Sudan, Improved decoding of Reed-Solomon and algebraic-geometry codes, IEEE Trans. Inform. Theory 45 (1999), 1757-1767.

[3] V. Guruswami and A. Vardy, Maximum-likelihood decoding of Reed-Solomon codes is NP-hard, IEEE Trans. Inform. Theory 51 (2005), 2249-2256.

[4] J. Li and D. Wan, On the subset sum problem over finite fields, Finite Fields Appls. 14 (2008), 911-929.

[5] Y. Li and D. Wan, On error distance of Reed-Solomon codes, Science in China Series A: Mathematics 51 (2008), 1982-1988.

[6] J.H. van Lint, Introduction to coding theory, Third Edition, Springer-Verlag, 1998.

[7] R.J. Mceliece, The theory of information and coding, Second Edition, Cambridge University Press, 2002.

[8] M. Sudan, Decoding of Reed-Solomon codes beyond the error-correction bound, J. Complexity 13 (1997), 180-193.

Mathematical College, Sichuan University, Chengdu 610064, P.R. China

E-mail address: eugen_woo@163.com

Yangtze Ctenter of Mathematics, Sichuan University, Chengdu 610064, P.R. China and Mathematical College, Sichuan University, Chengdu 610064, P.R. China

E-mail address: sfhong@scu.edu.cn, s-f.hong@tom.com, hongsf02@yahoo.com 